\title{
HARMONIC FUNCTIONS EXPRESSIBLE AS DIRICHLET SOLUTIONS ${ }^{1}$
}

\author{
Mitsuru NAKAI
}

\begin{abstract}
It is shown that any quasibounded harmonic function on any continuous domain can be expressed as a generalized solution of the harmonic Dirichlet problem on the domain with a resolutive boundary function on the boundary of the domain in the sense of Perron-Wiener-Brelot.
\end{abstract}

\section{Introduction}

The basic function space we consider is the linear space $H(R)$ of harmonic functions (i.e. $C^{2}$ solutions of the Laplace equation $\Delta u=0$ ) on a bounded subdomain $R$ of the Euclidean space $\boldsymbol{R}^{d}$ of dimension $d \geq 2$. We denote by $H P(R)$ the linear subspace of $H(R)$ consisting of harmonic functions $u$ on $R$ such that $|u|$ admit harmonic majorants on $R$ so that $H P(R)$ is the totality of harmonic functions which are differences of two positive harmonic functions on $R$. The significance of the class $H P(R)$ lies in the fact that it forms a vector lattice under the lattice operations $u \vee v$ (the least harmonic majorant of two harmonic functions $u$ and $v$ on $R$ ) and $u \wedge v$ (the greatest harmonic minorant of two harmonic functions $u$ and $v$ on $R$ ).

In this paper we mainly consider the relation between two linear subspaces of $H(R)$ mentioned below. The first is the space $H_{q b}(R)$ of quasibounded harmonic functions $u$ on $R$, where $u \in H(R)$ is quasibounded, by definition, if $u \in H P(R)$ and $(u \wedge \lambda) \vee(-\lambda)(\lambda>0)$ converges to $u$ almost uniformly on $R$ as $\lambda \uparrow \infty$. Therefore we have the inclusion relations $H B(R) \subset H_{q b}(R) \subset H P(R)$, where $H B(R)$ is the space of bounded harmonic functions on $R$. The second is the space $H_{d s}(R)$ of generalized Dirichlet solutions $H_{f}^{R}$ (cf. $\S 2$ below) on $R$ with resolutive boundary values $f$ on $\partial R$ in the sense of Perron-Wiener-Brelot.

It is readily seen that $H_{q b}(R) \supset H_{d s}(R)$ for every bounded domain $R$ (cf. $\S 3$ below) and there exists a domain $R$ such that the above inclusion relation is

\footnotetext{
${ }^{1} 1991$ Mathematics Subject Classification. Prımary 31B20, 31B25; Secondary 31B05.

This work was partly supported by Grant-in-Aid for Scientific Research, Nos. 09640180, 09640230, Japanese Ministry of Education, Science and Culture.

Recelved June 22, 1998.
} 
proper (cf. $\S 3$ below). If a harmonic function $u$ on $R$ is known to be expressed as $u=H_{f}^{R}$, then we are able to grasp, to a certain extent, the boundary behavior of $u$, which is very convenient in many instances. For this reason we are interested in finding the condition on $R$ for which the equality holds in the above inclusion relation; in other words, we wish to find the condition on $R$ under which the necessary condition that $u \in H_{q b}(R)$ for $u$ to be expressible as a Dirichlet solution is also sufficient. The purpose of this paper is to prove that if $R$ is a continuous domain (see $\$ 4$ below for the precise definition), then $H_{q b}(R)=$ $H_{d s}(R)$.

At this point we must remark that the class $H_{d s}(R)$ heavily depends upon the compactification of $R$ whereas the class $H_{q b}(R)$ is not. In this paper we are taking the Euclidean closure $\bar{R}$ of $R$ as its compactification which is the important point to be noted. If we adopt the Wiener or Martin compactification of $R$ and define the class $H_{d s}^{X}(R)$ accordingly (where $X=W$ or $M$ indicating that the class is considered with respect to the Wiener or Martin compactification), then we can obviously conclude the identity $H_{q b}(R)=H_{d s}^{X}(R)(X=W, M)$ at once. The Wiener compactification of $R$ is always different from $\bar{R}$ and hence this does not supply any information to our original problem. However there are cases where the Martin compactification of $R$ are known to be identical with $\bar{R}$, in which case our original problem is solved. But to find the condition that the Martin compactification coincides with $\bar{R}$ is probably harder than the original problem itself except for a few known cases. Therefore we will confine ourselves to the proper compactification $\bar{R}$ of $R$ and prove the following result.

THEOREM 1.1. Every quasibounded harmonic function on any continuous domain is expressible as a generalized Dirichlet solution on the domain of a resolutive boundary function on the boundary of the domain.

A continuous domain is roughly a domain which is expressed locally as one side of a graph of a continuous function in Cartesian or the polar coordinate. Examples of continuous domains are convex domains, star shaped domains, $C^{1}$ domains or more generally Lipschitz domains, certain Hölder domains, and so forth; closures of continuous domains may or may not be identical with their Martin compactifications; boundary points of continuous domain need not to be regular in general. Hence continuous domains may be considered to occupy a considerable portion of bounded domains $R$ of Carathéodory type, i.e. $\partial R=\partial \bar{R}$.

The proof of the above Theorem 1.1 will be given in $\$ 9$ after preparatory discussions in $\$ \$ 2-8$. In $\S 2$, certain inverse results are given deriving some properties of the boundary functions $f$ from the Dirichlet solutions $H_{f}^{R}$. The general inclusion relation $H_{d s}(R) \subset H_{q b}(R)$ and the occurrence of the strict inclusion relation $H_{d s}(R)<H_{q b}(R)$ are shown in $\S 3$. In $\$ 4$ a rather tedious description of continuous domains and some related facts are stated. Nevertheless the intuitive meaning of continuous domains should be clear. An old notion of Wiener algebra though not too popular is stated in $\$ 5$ just for the sake of convenience. The proof of Theorem 1.1 starts in essence from $\S 6$ in which the 
extremization of Wiener harmonized function on a subdomain to the whole region is discussed and the corresponding extremization of Dirichlet solution is considered in $\$ 7$. The essential use of the continuous domain property is made in $\$ 8$ and the main trick proving our theorem appears here. Summing up those results obtained in $\$ 6-8$, the proof is completed in the final $\$ 9$.

\section{Dirichlet solutions}

In addition to the class $H(R)$ of the space of harmonic functions on a domain $R$ in the Euclidean space $\boldsymbol{R}^{d}$ of dimension $d \geq 2$, we consider the class $\mathscr{S}(R)$ of superharmonic functions on $R$. For a class $\mathscr{F}$ of some functions we always set $\mathscr{F}^{+}:=\{f \in \mathscr{F}: f \geq 0\}$. We also consider the class $\mathscr{P}(R)$ of potentials $p$ on $R$ characterized by that $p \in \mathscr{S}(R)^{+}$and the greatest harmonic minorant of $p$ is zero on $R$. Thus we include 0 in $\mathscr{P}(R)$.

In this paper we consider the Dirichlet problem mainly for a bounded domain $R$ in $\boldsymbol{R}^{d}$ and a general boundary function $f$ on $\partial R$ with respect to the Laplace equation $\Delta u=0$. We follow the usual procedure of Perron-WienerBrelot (cf. e.g. [2, pp. 156-176]): we denote by $\overline{\mathscr{V}}_{f}^{R}$ the class of lower bounded $s \in \mathscr{S}(R)$ such that $\lim \inf _{x \in R, x \rightarrow y} s(x) \geq f(y)$ for every $y \in \partial R$; we set $\underline{\mathscr{V}}_{f}^{R}=$ $-\overline{\mathscr{V}}^{R}$; we denote by $\bar{H}_{f}^{R}\left(\underline{H}_{f}^{R}\right.$, resp.) the lower (upper, resp.) envelope of $\overline{\mathscr{V}}_{f}^{R}\left(\underline{\mathscr{V}}_{f}^{R}\right.$, resp.), which is either harmonic or identically $\pm \infty$; we see that $\bar{H}_{f}^{R} \geq$ $\underline{H}_{f}^{R}$ and $\bar{H}_{f}^{R}=-\underline{H}^{R}$ and, if $\bar{H}_{f}^{R}=\underline{H}_{f}^{R}$ is harmonic, then the common function is denoted by $H_{f}^{R}$ and $f$ is said to be resolutive; $H_{f}^{R}$ is referred to as the Dirichlet solution with the resolutive boundary function $f$.

We denote by $d \omega_{x}^{R}$ the harmonic measure on $\partial R$ characterized by $H_{f}^{R}(x)=$ $\int_{\partial R} f d \omega_{x}^{R}$ for each $x \in R$ and each $f \in C(\partial R)$. We fix a point $x_{0} \in R$ and use the simplified notation $d \omega=d \omega^{R}=d \omega_{x_{0}}^{R}$. There is a function $P(\cdot, x) \in L^{\infty}(\partial R, d \omega)$ (and actually a Borel function $P(\cdot, x)$ such that $c^{-1} \leq P(\cdot, x) \leq c d \omega$-a.e. on $\partial R$ with the Harnack constant $c$ determined by $x$ and $x_{0}$ and $R$ ) such that $d \omega_{x}^{R}=$ $P(\cdot, x) d \omega$ on $\partial R$. Then a function $f$ on $\partial R$ is resolutive if and only if $f \in$ $L^{1}(\partial R, d \omega)$ and in this case $H_{f}^{R}(x)=\int_{\partial R} f(y) P(y, x) d \omega(y)(x \in R)$.

We use the following fact which is an easy consequence of the definition of $\bar{H}_{f}^{R}$ and $\underline{H}_{f}^{R}$ : for any function $f$ on $\partial R$ for which $\bar{H}_{f}^{R}\left(\underline{H}_{f}^{R}\right.$, resp.) is harmonic there is a Borel function $g$ (and actually a decreasing (increasing, resp.) limit of lower (upper, resp.) semicontinuous functions) on $\partial R$ such that $f \leq g \leq$ $\left\|f ; L^{\infty}(\partial R, d \omega)\right\| \leq \infty\left(f \geq g \geq-\left\|f ; L^{\infty}(\partial R, d \omega)\right\| \geq-\infty\right.$, resp. $)$ on $\partial R$ with $\bar{H}_{f}^{R}=H_{g}^{R}\left(\underline{H}_{f}^{R}=H_{g}^{R}\right.$, resp.). In particular we see that for any resolutive function $f$ on $\partial R$ there is a Borel function $g$ on $\partial R$ with $|g| \leq \| f$; $L^{\infty}(\partial R, d \omega) \| \leq \infty$ on $\partial R$ and $g=f d \omega$-a.e. on $\partial R$ such that $H_{f}^{R}=H_{g}^{R}$.

As is well known and also easy to see, the operator $f \mapsto H_{f}^{R}$ from $L^{1}(\partial R, d \omega)$ into $H P(R)$ is positive and linear. Then the class $H_{d s}(R)$ introduced in Introduction is nothing but the image of the operator, i.e. $f \mapsto H_{f}^{R}$ : $L^{1}(\partial R, d \omega) \rightarrow H_{d s}(R)$ is a surjective linear operator. Less trivial is its injectiveness. This instantly follows from the following result. 
Proposition 2.1. If $\bar{H}_{f}^{R}$ and $\underline{H}_{f}^{R}$ are harmonic on $R$, then the inequalities

$$
\liminf _{x \in R, x \rightarrow y} \underline{H}_{f}^{R}(x) \leq f(y) \leq \limsup _{x \in R, x \rightarrow y} \bar{H}_{f}^{R}(x)
$$

hold for dw-a.e. $y$ in $\partial R$.

Proof. We take a $p \in \mathscr{S}(R)^{+}$such that $\bar{H}_{f}^{R}+\varepsilon p \in \overline{\mathscr{V}}_{f}^{R}$ for every positive number $\varepsilon$ (cf. e.g. [1, p. 21]). Let $F$ be the set of points $y \in \partial R$ such that $\liminf _{x \in R, x \rightarrow y} p(x)=+\infty$. Since $\varepsilon p \in \overline{\mathscr{V}}_{\chi_{F}}^{R}\left(\chi_{F}\right.$ being the characteristic function of $F$ on $\partial R$ ) for every $\varepsilon \geq 0$, we see that $F$ is of harmonic measure (i.e. $d \omega$ -measure) zero. Let $y \in \partial R \backslash F$. Then $\bar{H}_{f}^{R}+\varepsilon p \in \overline{\mathscr{V}}_{f}^{R}$ implies that

$$
f(y) \leq \liminf _{x \in R, x \rightarrow y}\left(\bar{H}_{f}^{R}(x)+\varepsilon p(x)\right) \leq \limsup _{x \in R, x \rightarrow y} \bar{H}_{f}^{R}(x)+\varepsilon \liminf _{x \in R, x \rightarrow y} p(x)
$$

for every $\varepsilon>0$. Since $0 \leq \liminf _{x \in R, x \rightarrow y} p(x)<\infty$, we conclude that the inequality of the right hand side of $(2.1)$ holds by letting $\varepsilon \downarrow 0$. To show the left hand side of (2.1) observe that $\bar{H}_{-f}^{R}$ is also harmonic since $\bar{H}_{-f}^{R}=-\underline{H}_{f}^{R}$ is harmonic by the assumption on $\underline{H}_{f}^{R}$. Therefore, by the same argument as above, we see that

$$
-f(y) \leq \limsup _{x \in R, x \rightarrow y} \bar{H}_{-f}^{R}(x)
$$

holds for $d \omega$-a.e. $y$ in $\partial R$, which is nothing but the validity of the left hand side of (2.1), and the proof is herewith complete.

For two functions $\varphi$ and $\psi$ we denote by $\varphi \cup \psi=\max (\varphi, \psi)$ and $\varphi \cap \psi=$ $\min (\varphi, \psi)$. One should not be confused by these with $u \vee v$ and $u \wedge v$ used only for harmonic functions $u$ and $v$. Since $H_{f}^{R}=H_{f \cup 0}^{R}-H_{(-f) \cup 0}^{R}$, we see that $H_{d s}(R) \subset H P(R)$ and actually $H_{d s}(R)$ is a vector sublattice of the vector lattice $H P(R)$. Of course $L^{1}(\partial R, d \omega)$ is a vector lattice under the lattice operations $U$ and $\cap$. Then we can conclude using (2.1) that $H_{d s}(R)$ is isomorphic to $L^{1}(\partial R, d \omega)$ as vector lattices. In fact, for example, in addition to the trivial implication from $f \geq 0$ on $\partial R$ to $H_{f}^{R} \geq 0$ on $R,(2.1)$ assures the inverse implication from $H_{f}^{R} \geq 0$ on $R$ to $f \geq 0$ on $\partial R$.

\section{Quasiboundedness}

In Introduction we introduced the class $H_{q b}(R)$ of quasibounded harmonic functions on $R$, which is also a vector sublattice of $H P(R)$. A function $u \in$ $H P(R)$ is said to be singular if $(u \wedge \lambda) \vee(-\lambda) \equiv 0$ on $R$ for any positive number $\lambda$. The totality of such functions is denoted by $H_{s}(R)$. Then we have the Parreau decomposition $H P(R)=H_{q b}(R) \oplus H_{s}(R)$ (the direct sum).

Take any $f \in L^{1}(\partial R, d \omega)$, which is equivalent to $f$ being resolutive. Then $\left(H_{f}^{R} \wedge \lambda\right) \vee(-\lambda)=\left(H_{f}^{R} \wedge H_{\lambda}^{R}\right) \vee H_{-\lambda}^{R}=H_{f \cap \lambda}^{R} \vee H_{-\lambda}^{R}=H_{(f \cap \lambda) \cup(-\lambda)}^{R}=\int_{\partial R}$ 
$(f(y) \cap \lambda) \cup(-\lambda) P(y, \cdot) d \omega(y)$, which, by the fact that $|(f \cap \lambda) \cup(-\lambda)| \leq|f|$ and $(f \cap \lambda) \cup(-\lambda) \rightarrow f$ as $\lambda \uparrow \infty$ on $R$ and by the Lebesgue dominated convergence theorem, converges to $\int_{\partial R} f(y) P(y, \cdot) d \omega(y)=H_{f}^{R}$ on $R$. This means that $H_{f}^{R} \in$ $H_{q b}(R)$. Thus we have established the following inclusion relation

$$
H_{d s}(R) \subset H_{q b}(R)
$$

for every bounded domain $R$. The Parreau decomposition with (3.1) assures that $H_{d s}(R) \cap H_{s}(R)=\{0\}$.

Next we exhibit a simple example of a domain $R$ for which the inclusion relation (3.1) is proper. We denote by $B$ the open unit ball in $\boldsymbol{R}^{d}$. Let $X=$ $B \backslash\left\{x=\left(x^{1}, \ldots, x^{d}\right): x^{1} \geq 0, x^{d}=0\right\}$. Then our claim is that

$$
H_{d s}(X)<H_{q b}(X),
$$

where $<$ means the proper inclusion: $\subset$ and $\neq$. We denote by $B^{+}=$ $\left\{x=\left(x^{\prime}, x^{d}\right) \in B: x^{d}>0\right\}$ and $B^{-}=\left\{x=\left(x^{\prime}, x^{d}\right) \in B: x^{d}<0\right\}$. Similarly we set $b^{+}=\left\{x=\left(x^{1}, \ldots, x^{d}\right) \in B: x^{1}>0, x^{d}=0\right\} \quad$ and $b^{-}=\left\{x=\left(x^{1}, \ldots, x^{d}\right) \in\right.$ $\left.B: x^{1}<0, x^{d}=0\right\}$. Let $u:=H_{\chi_{\bar{b}}^{B^{+}}}^{B^{+}}\left(\chi_{\overline{b^{+}}}\right.$being the characteristic function of $\overline{b^{+}}$on $\partial B^{+}$), which has boundary values zero on $\partial B^{+} \backslash \overline{b^{+}}$and in particular on $b^{-}$and 1 on $b^{+}$so that $u>0$ on $B^{+}$. By the reflection principle, $u$ can be continued to $X$ across $b^{-}$so as to satisfy $u \in H(X)$ and $u(\tilde{x})=-u(x)(x \in X)$, where $\tilde{x}=$ $\left(x^{\prime},-x^{d}\right)$ for $x=\left(x^{\prime}, x^{d}\right)$. Since it is bounded, we trivially have $u \in H_{q b}(X)$. We wish to show that $u \notin H_{d s}(X)$. Contrariwise suppose $u \in H_{d s}(X)$ so that there exists an $f \in L^{1}(\partial X, d \omega)$ with $u=H_{f}^{X}$ on $X$. By the boundary behavior of $u \mid B^{+}$we see that $u=0$ on $\partial B \backslash \overline{b^{+}}$. Hence $f=0$ on $\partial B \backslash \overline{b^{+}}$by Proposition 2.1. This means that $H_{f}^{X}$ is symmetric with respect to the plane $\left\{x=\left(x^{\prime}, x^{d}\right): x^{d}=0\right\}$. Then $u$ is symmetric (i.e. $\left.u(\tilde{x})=u(x)(x \in X)\right)$ and at the same time antisymmetric (i.e. $u(\tilde{x})=-u(x)(x \in X)$ ) which must imply $u=0$, which contradicts $u \mid B^{+}>0$.

In view of (3.1) and (3.2) we are naturally led to investigate the question when $H_{d s}(R)=H_{q b}(R)$ occurs. The example in (3.2) suggests that to have the identity there should not be too many boundary points of $R$ at which $R$ is not locally connected. For the rest of this paper we are concerned with such a sufficient condition.

\section{Continuous domains}

We call a coordinate system on $\boldsymbol{R}^{d}$ a Cartesian coordinate (polar coordinate, resp.) if it is obtained from the standard Cartesian coordinate $x=\left(x^{1}, \ldots, x^{d}\right)=$ $\left(x^{\prime}, x^{d}\right)$ (the standard polar coordinate $x=r \xi=(r, \xi)$ with $r \geq 0$ and $|\xi|=1$, resp.) by translation and rotation on $\boldsymbol{R}^{d}$.

For a Cartesian coordinate $x=\left(x^{1}, \ldots, x^{d}\right)=\left(x^{\prime}, x^{d}\right)$ we use the following notation: for any positive number $a>0$ we set

$$
\beta(a):=\left\{x^{\prime} \in \boldsymbol{R}^{d-1}:\left|x^{\prime}\right|:=\sqrt{\left(x^{1}\right)^{2}+\cdots+\left(x^{d-1}\right)^{2}}<a\right\}
$$


and, for any $a, b>0$ and any real number $c$ we consider a cylinder

$$
U(c ; a, b):=\left\{x=\left(x^{\prime}, x^{d}\right): x^{\prime} \in \beta(a),\left|x^{d}-c\right|<b\right\} .
$$

The point with coordinate $\left(0^{\prime}, c\right)$ will be referred to as the center of the cylinder $U(c ; a, b)$, where $0^{\prime}=(0, \ldots, 0)$ is the origin of $\boldsymbol{R}^{d-1}$ in this coordinate.

For a polar coordinate $x=r \xi=(r, \xi)$ we use the similar notation as above: for any $a \in(0,2)$ we set

$$
\beta(a):=\left\{\xi \in S^{d-1}:\left|\xi-e_{1}\right|<a\right\}
$$

with $S^{d-1}=\left\{\xi \in \boldsymbol{R}^{d}:|\xi|=1\right\}$ and $e_{1}=(1,0, \ldots, 0) \in S^{d-1}$ and, for any $0<a<$ 2 and $c>0$ and for any $b \in(0, c)$ we consider a sectorial ring

$$
U(c ; a, b):=\{x=r \xi: \xi \in \beta(a),|r-c|<b\},
$$

for which the point with coordinate $c e_{1}$ will be called its center.

Consider a domain $R$ in $\boldsymbol{R}^{d}$. A point $p \in \partial R$ is said to be a Cartesian graphic point for $R$ if there exists a Cartesian coordinate $x=\left(x^{\prime}, x^{d}\right)$ for which the coordinate of $p$ is $\left(0^{\prime}, c\right)(c>0)$ and there exist two positive numbers $a$ and $b$ and a continuous function $x^{d}=\varphi\left(x^{\prime}\right)$ defined on $\beta(a)$ such that $\sup _{\beta(a)}|\varphi-c|<b$ and

$$
U(c ; a, b) \cap R=\left\{x=\left(x^{\prime}, x^{d}\right): x^{\prime} \in \beta(a), c-b<x^{d}<\varphi\left(x^{\prime}\right)\right\}
$$

and at the same time we have

$$
U(c ; a, b) \cap \partial R=\left\{x=\left(x^{\prime}, x^{d}\right): x^{\prime} \in \beta(a), x^{d}=\varphi\left(x^{\prime}\right)\right\} .
$$

In this case the neighborhood $U(c ; a, b)$ of $p$ is said to be an admissible neighborhood of $p$ and the function $x^{d}=\varphi\left(x^{\prime}\right)$ is called the local representing function of $R$ (or $\partial R$ ) associated with $U(c ; a, b)$.

A point $p \in \partial R$ is said to be a polar graphic point for $R$ if there exists a polar coordinate $x=r \xi$ for which the coordinate of $p$ is $c e_{1}(c>0)$ and there exist two numbers $0<a<2$ and $0<b<c$ and a continuous function $r=\varphi(\xi)>0$ defined on $\beta(a)$ such that $\sup _{\beta(a)}|\varphi-c|<b$ and

$$
U(c ; a, b) \cap R=\{x=r \xi: \xi \in \beta(a), c-b<r<\varphi(\xi)\}
$$

and simultaneously it holds that

$$
U(c ; a, b) \cap \partial R=\{x=r \xi: \xi \in \beta(a), r=\varphi(\xi)\} .
$$

In such a case the neighborhood $U(c ; a, b)$ of $p$ is referred to as an admissible neighborhood at $p$ and the function $r=\varphi(\xi)$ is said to be the local representing function of $R$ (or $\partial R$ ) associated with $U(c ; a, b)$.

In either of the above two parallel definitions of graphic points we assumed that $\varphi$ is continuous. However this is not a thing to be assumed but the consequence of the very definitions: if we simply assume that $\varphi$ is merely a single valued function in either of the above two definitions, then we can prove that $\varphi$ must be automatically continuous. It should also be noted that there is a plenty 
of examples of $R$ such that $\partial R$ contains points which are Cartesian (polar, resp.) graphic points but not polar (Cartesian, resp.) graphic points.

Finally a point $p$ in $\partial R$ is referred to simply as a graphic point for $R$ if $p$ is either a Cartesian graphic point or a polar graphic point. A bounded domain $R$ in $\boldsymbol{R}^{d}$ will be called a continuous domain (cf. e.g. [3]) if every boundary point of $R$ is a graphic point for $R$. It may happen for continuous domain $R$ that there are two points in $\partial R$ one of which is a Cartesian graphic point for $R$ and another of which is a polar graphic point.

For any continuous domain $R$ we can find a finite open covering $\left\{U_{i}\right\}_{0 \leq l \leq l}$ of $\bar{R}$ such that each $U_{i}(1 \leq i \leq l)$ is an admissible neighborhood $U\left(c_{i} ; a_{i}, b_{i}\right)$ whose center is a point in $\partial R$ and $\bar{U}_{0} \subset R$. Such a covering $\left\{U_{i}\right\}_{0 \leq l \leq l}$ is referred to as an admissible covering of $\bar{R}$. A partition of unity $\left\{\varphi_{i}\right\}_{0 \leq \imath \leq m}$ on $\bar{R}$ is said to be an admissible partition of unity on $\bar{R}$ subordinate to an admissible covering $\left\{U_{i}\right\}_{0 \leq l \leq l}$ of $\bar{R}$ if the following three conditions are satisfied: each $\varphi_{i} \in C_{0}^{\infty}\left(\boldsymbol{R}^{d}\right)(0 \leq i \leq m) ;\left\{\varphi_{i}\right\}_{0 \leq l \leq m}$ is subordinate to the admissible covering $\left\{U_{i}\right\}_{0 \leq l \leq l}$ of $\bar{R}$ such that the support spt $\varphi_{0}$ of $\varphi_{0}$ is contained in $U_{0}$ and for each $1 \leq i \leq m$ there is a $1 \leq j \leq l$ with $\operatorname{spt} \varphi_{i} \subset U_{j} ; \sum_{l=1}^{m} \varphi_{i} \equiv 1$ on a neighborhood of $\partial R$ and $\sum_{t=0}^{m} \varphi_{i} \equiv 1$ on a neighborhood of $\bar{R}$. The existence of such an admissible partition of unity on $\bar{R}$ can be easily shown.

\section{Wiener algebra}

Consider a real valued function $f$ on a bounded domain $R$ in $\boldsymbol{R}^{d}$. We denote by $\overline{\mathscr{W}}_{f}^{R}$ the class of functions $s \in \mathscr{S}(R)$ having compact subsets $K_{s} \subset R$ such that $s \geq f$ on $R \backslash K_{s}$. We denote by $\bar{h}_{f}^{R}$ the lower envelope of the class $\overline{\mathscr{W}}_{f}^{R}$ and set $\underline{h}_{f}^{R}:=-\bar{h}_{-f}^{R}$. The functions $\bar{h}_{f}^{R}$ and $\underline{h}_{f}^{R}$ are either harmonic or identically $\pm \infty$ on $R$ and satisfy $\bar{h}_{f}^{R} \geq \underline{h}_{f}^{R}$ on $R$. If $\bar{h}_{f}^{R}=\underline{h}_{f}^{R}$ on $R$ and the common function is harmonic on $R$, then we say that $f$ is harmonizable on $R$ and we denote the common function by $h_{f}^{R}$ (cf. [1, pp. 54-55]).

Still assuming $R$ to be bounded we denote by $N(R)$ the class of bounded continuous functions $f$ on $R$ which are harmonizable on every subdomain of $R$. It is known that $N(R)$ forms a Banach algebra having the multiplicative identity 1 equipped with the supremum norm and also $N(R)$ is a vector lattice under the lattice operations $U$ and $\cap$ (cf. [4, pp. 223-227]). The most important point is that $N(R)$ is an algebra and complete with respect to the uniform convergence. The operator $f \mapsto h_{f}^{R}: N(R) \rightarrow H B(R)$ is seen to be a homomorphism as vector lattices. Thus we have e.g. $h_{f \cap g}^{R}=h_{f}^{R} \wedge h_{g}^{R}$ on $R$.

We denote by $C_{b}(R)$ the class of bounded continuous functions on $R$. It is not difficult to see that any function $s \in \mathscr{S}(R) \cap C_{b}(R)$ is harmonizable on any subdomain $V$ of $R$. In fact, let $u$ be the greatest harmonic minorant of $s$ and $\left(V_{l}\right)_{i \geq 1}$ be an exhaustion of $V$ by regular domains. Then $\hat{R}_{s}^{V \backslash V_{l}, V} \downarrow u$ as $i \uparrow \infty$, where $\hat{R}_{s}^{V \backslash V_{l}, V}$ is the balayage of $s$ over the set $V \backslash V_{l}$ with respect to the domain $V$. Since $\hat{R}_{s}^{V \backslash V_{l}, V} \in \overline{\mathscr{W}}_{s}^{V}$, we see that $\hat{R}_{s}^{V \backslash V_{l}, V} \geq \bar{h}_{s}^{V} \geq \underline{h}_{s}^{V} \geq u$ on $V$, which 
implies that $\bar{h}_{s}^{V}=\underline{h}_{s}^{V}=u$ on $V$. Hence we see that

$$
\mathscr{S}(R) \cap C_{b}(R) \subset N(R) .
$$

Let $f \in C\left(\boldsymbol{R}^{d}\right)$ and $S$ be a bounded domain with $\bar{R} \subset S$. As is well known there are sequences $\left(s_{i}^{\prime}\right)_{i \geq 1}$ and $\left(s_{i}^{\prime \prime}\right)_{i \geq 1}$ in $\mathscr{S}(S) \cap C(S)$ such that $\left(s_{i}\right)_{i \geq 1}$ with $s_{i}=s_{i}^{\prime}-s_{i}^{\prime \prime}$ converges uniformly to $f$ on $\bar{R}$. By (5.1), $s_{i}^{\prime}$ and $s_{i}^{\prime \prime}$ and hence $s_{i}$ belong to $N(R)$. By the completeness of $N(R)$ in the uniform convergence, we can conclude that $f \in N(R)$. Hence we have the following relation:

$$
C\left(\boldsymbol{R}^{d}\right) \mid R \subset N(R) .
$$

We will use (5.2) in the following fashion. Let $u \in H B(R)$ ad $\varphi \in C_{0}^{\infty}\left(\boldsymbol{R}^{d}\right)$. It is obvious that $u \in N(R)$, and by (5.2), $\varphi \in N(R)$. Hence the closedness of $N(R)$ under multiplication assures that $\varphi u \in N(R)$.

\section{Wiener extremization}

Fix an admissible neighborhood $U(c ; a, b)$ with a center $p_{0}$ in the boundary $\partial R$ of a continuous domain $R$ and set $V:=R \cap U(c ; a, b)$. According as $U(c ; a, b)$ is considered with respect to a Cartesian coordinate or polar coordinate, we let the coordinate of the center $p_{0}$ be $\left(0^{\prime}, c\right)$ in the Cartesian coordinate or $c e_{1}$ in the polar coordinate in question. We will prove the following result.

LEMMA 6.1. If $v \in N(R)^{+}$satisfies $v=0$ on $R \backslash U\left(c ; a^{\prime}, b^{\prime}\right)\left(0<a^{\prime}<a\right.$, $\left.0<b^{\prime}<b\right)$, then $h_{v}^{V}$ can be continuously extended to $R$ by setting $h_{v}^{V}=0$ on $R \backslash V$ and the extended $h_{v}^{V}$ is subharmonic on $R$ and has boundary values zero at each point of $\partial R \backslash U(c ; a, b)$. Moreover the extremization of $h_{v}^{V}$ on $V$ to $R$ is $h_{v}^{R}$ in the sense that

$$
h_{v}^{R}-h_{v}^{V} \in \mathscr{P}(R),
$$

where $h_{v}^{V}$ is considered to be the above extended subharmonic function on $R$.

Proof. For simplicity we set $A:=U(c ; a, b)$ and $C:=U\left(c ; a^{\prime}, b^{\prime}\right)$ so that $V=R \cap A$. Observe that $(6.1)$ is equivalent to $0=\mathrm{GHM}\left[h_{v}^{R}-h_{v}^{V}\right]=h_{v}^{R}-$ $\operatorname{LHM}\left[h_{v}^{V}\right]$, where GHM $[s](\operatorname{LHM}[s]$, resp.) means the greatest harmonic minorant (the least harmonic majorant, resp.) of a superharmonic (subharmonic, resp.) function $s$ on $R$. Thus we only have to show that

$$
\operatorname{LHM}\left[h_{v}^{V}\right]=h_{v}^{R} .
$$

Consider $t:=\hat{R}_{1}^{\bar{C} \cap R, V}$, where the term on the right means the balayage of the constant function 1 on $\bar{C} \cap R$ relative to the domain $V$ so that $t$ is the harmonic measure of $\bar{C} \cap R$ on the domain $V \backslash \bar{C}$. By the cone condition every point of $\partial V \backslash A$ is regular and thus $t$ has boundary values zero at every point in $\partial V \backslash A$. The delicate points are those in $\partial A \cap \partial R$ but this set is contained in $\partial V \backslash A$. Let $\gamma:=\sup _{R} v$. Clearly $\gamma t \in \overline{\mathscr{W}}_{v}^{V}$ and hence $0 \leq h_{v}^{V} \leq \gamma t$ so that $h_{v}^{V}$ 
also has boundary values zero at every point in $\partial V \backslash A$. Hence $h_{v}^{V}$ can be continuously extended to $R$ by setting $h_{v}^{V}=0$ on $R \backslash V$ and the extended $h_{v}^{V}$ has boundary values zero at each point of $\partial R \backslash U(c ; a, b)$. Hereafter we use the notation $h_{v}^{V}$ in this extended sense. Clearly $h_{v}^{V} \in-\mathscr{S}(R)$, i.e. $h_{v}^{V}$ is subharmonic on $R$.

For the proof of $(6.2)$ we need a function $p \in \mathscr{S}(R)^{+}$with the following property:

$$
h_{v}^{V}+\frac{1}{m} p \in \overline{\mathscr{W}}_{v}^{V} \quad(m \in N)
$$

where $N$ is the set of positive integers. We now explain the procedure of the construction of $p$ and show it actually satisfies (6.3).

Fix an arbitrary point $x_{0} \in V$ and choose $u_{i} \in \bar{W}_{v}^{V}$ such that $u_{i}\left(x_{0}\right)-$ $h_{v}^{V}\left(x_{0}\right)<2^{-l}$ for each $i \in N$ and set $q:=\sum_{l=1}^{\infty}\left(u_{i}-h_{v}^{V}\right)$, which belongs to $\mathscr{S}(V)^{+}$. Modify $q$ to $u:=\hat{R}_{q}^{\bar{C} \cap R, V} \in \mathscr{S}(V)^{+}$. Again by the cone condition, $u$ can be continuously continued to $\bar{R} \cap \partial A$ on which $u$ takes the value 0 . In view of this if we take $a^{\prime}<a^{\prime \prime}<a$ and $b^{\prime}<b^{\prime \prime}<b$ sufficiently close to $a$ and $b$, then

$$
\sup _{R \cap \partial B} u<\infty
$$

where $B:=U\left(c ; a^{\prime \prime}, b^{\prime \prime}\right)$. Next take $w:=\hat{R}_{1}^{\bar{B} \cap R, R}$, the harmonic measure of $\bar{B} \cap R$ on the domain $R \backslash \bar{B}$, i.e. $w \in \mathscr{S}(R)^{+} \cap C(R) \cap H(R \backslash \bar{B})$ with $w=1$ on $\bar{B} \cap R$. Take a large ball $D$ such that $\bar{R} \subset D$. Then $w^{\prime}:=\hat{R}_{1}^{\bar{B}, D}$ takes the values less than 1 on $\partial A$. Since $0<w \leq w^{\prime}$ on $R$ we see that

$$
\sup _{R \cap \partial A} w<1 \text {. }
$$

Therefore if we choose a positive number $M^{\prime}$ sufficiently large, then the following inequality holds:

$$
\sup _{R \cap \partial A} w<1-\frac{1}{M^{\prime}} \sup _{R \cap \partial B} u \text {. }
$$

We fix such an $M^{\prime}$. Then we can find a positive number $M$ such that

$$
\frac{M}{M^{\prime}} \in\left(\sup _{R \cap \partial A} w, 1-\frac{1}{M^{\prime}} \sup _{R \cap \partial B} u\right) .
$$

We also fix such an $M$. Then, since $M^{\prime} \sup _{R \cap \partial A} w<M=u+M$ on $R \cap \partial A$, we see that

$$
u+M>M^{\prime} w \text { on } R \cap \partial A .
$$

Similarly, since $\sup _{R \cap \partial B} u+M<M^{\prime}=M^{\prime} w$ on $R \cap \partial B$, we obtain

$$
u+M<M^{\prime} w \text { on } R \cap \partial B .
$$

Using the above last two displayed inequalities we can easily see that the function 
$p$ on $R$ defined by

$$
p:= \begin{cases}u+M & \text { on } B \cap R \\ \min \left(u+M, M^{\prime} w\right) & \text { on }(A \backslash B) \cap R \\ M^{\prime} w & \text { on } R \backslash A\end{cases}
$$

is superharmonic on $R$, i.e. $p \in \mathscr{S}(R)^{+}$.

Let $K_{l}$ be a compact set in $V$ such that $u_{i} \geq v$ on $V \backslash K_{l}$ for each $1 \leq i \leq m$ for an arbitrarily fixed $m$ and set $K:=\bigcup_{l=1}^{m} K_{l}$, which is a compact set in $V$. Then $(1 / m) \sum_{l=1}^{m} u_{i} \geq v$ on $V \backslash K$. Hence, on $(C \cap R) \backslash K$,

$$
h_{v}^{V}+\frac{1}{m} p=h_{v}^{V}+\frac{1}{m}(u+M)>h_{v}^{V}+\frac{1}{m} q>h_{v}^{V}+\frac{1}{m} \sum_{l=1}^{m}\left(u_{i}-h_{v}^{V}\right)=\frac{1}{m} \sum_{l=1}^{m} u_{i} \geq v
$$

i.e. $h_{v}^{V}+(1 / m) p \geq v$ on $(C \cap R) \backslash K$. This is also true on $V \backslash C$ since $v=0$ there. Thus $h_{v}^{V}+(1 / m) p \geq v$ on $V \backslash K$, which implies (6.3).

Choose an arbitrary $u \in \overline{\mathscr{W}}_{v}^{R}$ so that there is a compact set $K \subset R$ such that $u \geq v$ on $R \backslash K$. By $v=0$ on $R \backslash C, u \geq v$ holds on $R \backslash K \cap \bar{B}$ and a fortiori on $V \backslash K \cap \bar{B}$ so that we have $u \mid V \in \overline{\mathscr{W}}_{v}^{V}$. Thus $u \geq h_{v}^{V}$ holds on $V$ and naturally on $R$, which implies that $h_{v}^{R} \geq h_{v}^{V}$ and in turn $\operatorname{LHM}\left[h_{v}^{V}\right] \leq h_{v}^{R}$. Conversely, take an arbitrary $u \in \mathscr{S}(R)^{+}$such that $h_{v}^{V} \leq u$ on $R$. By (6.3), there is a compact set $K \subset V$ such that $h_{v}^{V}+(1 / m) p \geq v$ on $V \backslash K$. The last inequality is trivially true on $R \backslash V$ and therefore

$$
u+\frac{1}{m} p \geq h_{v}^{V}+\frac{1}{m} p \geq v
$$

on $R \backslash K$, i.e. $u+(1 / m) p \in \overline{\mathscr{W}}_{v}^{R}$ and thus $u+(1 / m) p \geq h_{v}^{R}$ on $R$. On letting $m \uparrow \infty$ and then on taking the infimum with respect to $u \in \mathscr{S}(R)$ with $u \geq h_{v}^{V}$ on $R$, we can conclude that $\operatorname{LHM}\left[h_{v}^{V}\right] \geq h_{v}^{R}$, which proves (6.2).

\section{Dirichlet extremization}

Let $U(c ; a, b)$ and $V:=R \cap U(c ; a, b)$ be as in $\S 6$. We set $\alpha:=\overline{U(c ; a, b)} \cap$ $\partial R$, which is a subset of $\partial V$ and at the same time a subset of $\partial R$. Let $f$ be a bounded nonnegative Borel function on $\alpha$ vanishing continuously at each point of $\partial U(c ; a, b) \cap \partial R$. We extend $f$ to $\boldsymbol{R}^{d}$ by setting $f=0$ on $\boldsymbol{R}^{d} \backslash \alpha$. Hence if we write $H_{f}^{V}\left(H_{f}^{R}\right.$, resp.), then the boundary function $f$ is understood to be $f \mid \partial V$ $(f \mid \partial R$, resp.). The following result is a counterpart to (6.1) but its proof this time is easy and immediate.

Lemma 7.1. The function $H_{f}^{V}$ on $V$ can be continuously extended to $R$ by setting $H_{f}^{V}=0$ on $R \backslash V$ and thus extended $H_{f}^{V}$ is subharmonic on $R$. Moreover 
the extremization of $H_{f}^{V}$ on $V$ to $R$ is $H_{f}^{R}$ in the sense that

$$
H_{f}^{R}-H_{f}^{V} \in \mathscr{P}(R),
$$

where $H_{f}^{V}$ is understood to be the extended subharmonic function on $R$.

Proof. By the cone condition every point in $\partial U(c ; a, b) \cap \bar{R}$ is regular. Since $f=0$ on $\partial V \backslash \alpha$, we see that $H_{f}^{V}$ has boundary values 0 on $\partial V \backslash \alpha$. Thus if we extend $H_{f}^{V}$ to $R$ by setting $H_{f}^{V}=0$ on $R \backslash V$, then it is continuous on $R$ and easily seen to be subharmonic on $R$.

To see (7.1) we take a Borel function $\varphi$ on $\partial V$ defined by $\varphi=0$ on $\alpha$ and $\varphi=H_{f}^{R}$ on $\partial V \backslash \alpha$. We first note that

$$
H_{f}^{R} \mid V=H_{f+\varphi}^{V}=H_{f}^{V}+H_{\varphi}^{V} .
$$

In fact, take an arbitrary $s \in \overline{\mathscr{V}}_{f}^{R}$. Since $s \geq H_{f}^{R}$ on $V$, we have $s \in \overline{\mathscr{V}}_{f+\varphi}^{V}$ and a fortiori $H_{f}^{R} \mid V \geq H_{f+\varphi}^{V}$. Similarly $H_{-f}^{R} \mid V \geq H_{-f-\varphi}^{V}$, which means $H_{f}^{R} \mid V \leq$ $H_{f+\varphi}^{V}$. Thus we have deduced the above displayed identity. Note here that the sign of $f$ is immaterial in the above argument so that considering $-f$ in addition to $f$ is allowable.

We extend $H_{f}^{V}$ and $H_{\varphi}^{V}$ to $R$ by setting $H_{f}^{V}=0$ and $H_{\varphi}^{V}=H_{f}^{R}$ on $R \backslash V$. Then by the above displayed identity we still have $H_{f}^{R}=H_{f}^{V}+H_{\varphi}^{V}$ on $R$ so that

$$
H_{f}^{R}-H_{f}^{V}=H_{\varphi}^{V}
$$

on $R$. To deduce (7.1) we only have to show that the extended $H_{\varphi}^{V}$ is a potential on $R$. Since every point in $R \cap \partial V=\partial V \backslash \alpha$ is regular with respect to $V$, the extended $H_{\varphi}^{V} \in C(R)$. Since $H_{f}^{R} \geq H_{\varphi}^{V} \geq 0$ and $f$ vanishes continuously at each point of $\partial U(c ; a, b) \cap \partial R$, we see that $H_{\varphi}^{V} \in \mathscr{S}(R)$ and $H_{\varphi}^{V}$ has boundary values zero at every regular point of $\partial R$. This with the boundedness of $H_{\varphi}^{V}$ assures that it is a potential.

\section{Construction of boundary function}

Fix an admissible neighborhood $U(c ; a, b)$ with a center $p$ in the boundary $\partial R$ of a continuous domain $R$ and set $V:=R \cap U(c ; a, b)$. According to whether $p$ is a Cartesian or polar graphic point, we let the coordinate of the center $p$ be $\left(0^{\prime}, c\right)$ in the Cartesian coordinate or $c e_{1}$ in the polar coordinate in question. We now prove the following result.

LEMMA 8.1. If $v$ is a bounded nonnegative harmonic function on $V$ having boundary values zero on $\partial V \backslash U(c ; a, b)$, then there exists a bounded nonnegative Borel function $f$ on $\partial V$ vanishing continuously at each point of $\partial V \backslash U(c ; a, b)$ such that

$$
v(x)=H_{f}^{V}(x) \quad(x \in V) .
$$


Proof. Without loss of generality we may assume that $0 \leq v \leq 1$ on $V$. There is a $\gamma \in(0, b)$ such that even $U(c ; a, b-\gamma)$ is admissible. We now define $V_{l}$ and $v_{i}$ for $i \geq i_{0}$ separately according as $p$ is a Cartesian graphic point or $p$ is a polar graphic point.

In the case $p$ is a Cartesian graphic point we set $i_{0}:=1+[1 / \gamma]$, where $[\cdot]$ is the Gaussian symbol. For each $i \geq i_{0}$ we set $V_{l}:=\left\{x+(1 / i) e_{d}: x \in V\right\}$ where $e_{d}=(0, \ldots, 0,1) \in \boldsymbol{R}^{d}$. Consider a function $v_{i} \in H\left(V_{l} \cap R\right) \cap C(\bar{V})$ given by $v_{i}(x):=v\left(x-(1 / i) e_{d}\right)$ for $x \in V_{l} \cap R$ and $v_{i}(x)=0$ for $x \in \bar{V} \backslash V_{l}$. In the case $p$ is a polar graphic point we set $i_{0}:=1+[(c-b) / \gamma]$. For each $i \geq i_{0}$ we set $V_{l}:=\{(1+(1 / i)) x: x \in V\}$. Consider a function $v_{i} \in H\left(V_{l} \cap R\right) \cap C(\bar{V})$ given by $v_{i}(x):=v\left((1+(1 / i))^{-1} x\right)$ for $x \in V_{l} \cap R$ and $v_{i}(x)=0$ for $x \in \bar{V} \backslash V_{l}$. In either case we set $w_{i}:=H_{f_{t}}^{V}$, where $f_{l}=v_{i} \mid \partial V \in C(\partial V)$ and satisfies $0 \leq f_{l} \leq 1$ on $\partial V$.

We denote by $d \omega_{x}^{V}$ the harmonic measure on $\partial V$ evaluated at $x \in V$; fixing an arbitrary point $x_{0} \in V$ we set $d \omega=d \omega_{x_{0}}^{V}$; there is a function $P(\cdot, x) \in$ $L^{\infty}(\partial V, d \omega)$ such that $d \omega_{x}^{V}=P(\cdot, x) d \omega$ (cf. $\left.\S 2\right)$. We denote by $\left(L^{1}(\partial V, d \omega)\right)^{*}$ the dual space of $L^{1}(\partial V, d \omega)$ which is $L^{\infty}(\partial V, d \omega)$. By the Alaoglu theorem, the closed unit ball $B$ in $L^{\infty}(\partial V, d \omega)=\left(L^{1}(\partial V, d \omega)\right)^{*}$ is weakly * compact, which is characterized by the fact that any generalized sequence in $B$ has a cluster point in $B$. Hence the particular generalized sequence $\left(f_{l}\right)_{i \geq l_{0}}$ in $B$ has a cluster point $f^{\prime}$ in $B$. We will show that

$$
v(x)=H_{f^{\prime}}^{V}(x) \quad(x \in V) .
$$

For this purpose we only have to show that

$$
\left|v(x)-H_{f^{\prime}}^{V}(x)\right|<\varepsilon
$$

for any positive number $\varepsilon>0$ and any point $x \in V$ chosen arbitrarily and then fixed in advance.

If $i \geq i_{0}$ is sufficiently large, then $x \in V_{l} \cap R$ and hence $v_{i}(x)-v(x)=$ $v\left(x-(1 / i) e_{d}\right)-v(x)$ or $v\left((1+(1 / i))^{-1} x\right)-v(x)$, according as $p$ is a Cartesian or polar graphic point, tends to zero as $i \uparrow \infty$ by the continuity of $v$ on $V$. Therefore there exists a number $j_{1}>i_{0}$ such that

$$
\left|v_{i}(x)-v(x)\right|<\frac{\varepsilon}{3} \quad\left(i \geq j_{1}\right) .
$$

We introduce an auxiliary function $w:=H_{\varphi}^{V}$, where the boundary function $\varphi$ on $\partial V$ is given by $\varphi \mid R \cap \partial V=0$ and $\varphi \mid \bar{V} \cap \partial R=1$. Then $w$ has boundary values zero on $R \cap \partial V$ and one at every regular point in $\partial V \backslash \overline{R \cap \partial V}$. Clearly $0 \leq w \leq 1$ on $V$. Choose a sufficiently large ball $B(p, \rho):=\{x:|x-p|<\rho\} \supset \bar{R}$ and a function $q \in H(B(p, \rho) \backslash \partial R \cap \partial V)^{+}$such that $q$ has boundary values 0 on $\partial B(p, \rho)$ and $+\infty$ at each irregular point of $\partial R \cap \partial V$ with respect to $V$. Examining the boundary values of the harmonic function $w-w_{i}+\delta q$ on $V$ on $\partial V$ for an arbitrary positive number $\delta$ we see that $w+\delta q \geq w_{i}$ on $V$ so that $w \geq$ $w_{i}$ on $V$ by letting $\delta \downarrow 0$. In particular $0 \leq w_{i} \leq \sup _{\bar{V} \backslash V_{l}} w$ on $\bar{V} \backslash V_{l}$. Once again 
by examining the boundary values of the harmonic function $w_{i}-v_{i}-\delta q-$ $\sup _{\bar{V} \backslash V_{l}} w$ on $V_{l} \cap R$ on $\partial\left(V_{l} \cap R\right)$ for an arbitrary positive number $\delta$, we see it is nonpositive on $V_{l} \cap R$ and then on $V$ so that, by letting $\delta \downarrow 0$,

$$
0 \leq w_{i}-v_{i} \leq \sup _{\bar{V} \backslash V_{\imath}} w
$$

on $V$. The rightmost term of the above tends to zero as $i \uparrow \infty$. Therefore we can find a number $j_{2}>i_{0}$ such that

$$
\left|w_{i}(x)-v_{i}(x)\right|<\frac{\varepsilon}{3} \quad\left(i \geq j_{2}\right)
$$

Finally, since $f^{\prime}$ is a cluster point of the generalized sequence $\left(f_{l}\right)_{i \geq t_{0}}$ in the dual space $\left(L^{1}(\partial V, d \omega)\right)^{*}=L^{\infty}(\partial V, d \omega)$, for the number $j_{3}:=\max \left(j_{1}, j_{2}\right)>i_{0}$, there is a number $i_{1}>j_{3}$ such that $f_{l}$ is contained in the given neighborhood of $f^{\prime}$ in the dual space determined by $P(\cdot, x) \in L^{1}(\partial V, d \omega)$ and the positive number $\varepsilon / 3$, i.e.

$$
\left|\int_{\partial V} P(y, x) f_{l_{1}}(y) d \omega(y)-\int_{\partial V} P(y, x) f^{\prime}(y) d \omega(y)\right|<\frac{\varepsilon}{3},
$$

which means that

$$
\left|w_{i_{1}}(x)-H_{f^{\prime}}^{V}(x)\right|<\frac{\varepsilon}{3}
$$

Since $i_{1}>j_{1}$ and $j_{2}$, from (8.4) with $i=i_{1}$, (8.5) with $i=i_{1}$, and (8.6), the desired relation (8.3) follows so that we can conclude (8.2). We can find a Borel function $f^{\prime \prime}$ with $0 \leq f^{\prime \prime} \leq 1$ on $\partial V$ such that $v=H_{f^{\prime}}^{V}=H_{f^{\prime \prime}}^{V}$ on $V$ (cf. §2). Since $v$ has boundary values zero on $R \cap \partial V$, by Proposition 2.1, we can replace $f^{\prime \prime}$ by $f^{\prime \prime \prime}:=f^{\prime \prime} \chi_{\bar{V} \cap \partial R}\left(\chi_{\bar{V} \cap \partial R}\right.$ being the characteristic function of $\bar{V} \cap \partial R$ on $\left.\partial V\right)$, which is still a bounded nonnegative Borel function on $\partial V$ (since $\bar{V} \cap \partial R$ is a Borel subset in $\partial V$ ) vanishing continuously on $R \cap \partial V$ and satisfies $v=H_{f^{\prime \prime}}^{V^{\prime \prime}}=H_{f^{\prime \prime \prime} \text {. on }}^{V}$ $V$. Finally let $\beta:=\partial U(c ; a, b) \cap \partial R$ and $\beta(t)\left(\beta^{\prime}(t)\right.$, resp.) be the set of points $x \in \partial V \quad(x \in V$, resp. $)$ such that there is points $y_{x} \in \beta$ with $\left|x-y_{x}\right|<t$ for a positive number $t$. Since $v$ vanishes continuously at each point of $\beta$, there is a strictly decreasing sequence $\left(t_{t}\right)_{i \geq 1}$ of positive numbers $t_{i} \downarrow 0(i \uparrow \infty)$ such that $s_{i}:=\sup _{\beta^{\prime}\left(t_{i}\right)} v \downarrow 0(i \uparrow \infty)$. By $v=H_{f^{\prime \prime \prime}}^{V}$ on $V$ and by Proposition 2.1, we see that $0 \leq f^{\prime \prime \prime} \leq s_{i} d \omega$-a.e. on $\beta\left(t_{i}\right)$ and hence on $\beta\left(t_{i}\right) \backslash \beta\left(t_{i+1}\right)(i \in N)$. Hence if we replace $f^{\prime \prime \prime}$ by

$$
f:=\sum_{i=1}^{\infty}\left(f^{\prime \prime \prime} \cap s_{i}\right) \chi_{\beta\left(t_{i}\right) \backslash \beta\left(t_{i+1}\right)}+0 \cdot \chi_{\beta}+f^{\prime \prime \prime} \chi_{\partial V \bigvee \beta\left(t_{1}\right)},
$$

then we still can conclude that $f$ is a bounded nonnegative Borel function on $\partial V$ and $v=H_{f^{\prime \prime \prime}}^{V}=H_{f}^{V}$ on $V$ and moreover $f$ vanishes continuously on the set $\partial V \backslash$ $U(c ; a, b)$ and especially on $\beta=\partial U(c ; a, b) \cap \partial R$. 


\section{Proof of Theorem 1.1}

Given a $u \in H_{q b}(R)$ for a continuous domain $R$ and we are to find an $f \in$ $L^{1}(\partial R, d \omega)$, or equivalently a resolutive boundary function $f$ on $\partial R$, such that

$$
u=H_{f}^{R} \text { on } R,
$$

where $d \omega=d \omega_{x_{0}}^{R}$ for a fixed $x_{0} \in R$. First we remark that we may and thus we will assume that $0 \leq u \leq 1$ on $R$ in proving (9.1). In fact, since $H_{q b}(R)$ is a vector lattice, a general $u \in H_{q b}(R)$ can be expressed as $u=u^{+}-u^{-}\left(u^{+}=u \vee 0\right.$, $\left.u^{-}=-u \wedge 0\right)$. If $(9.1)$ holds for functions in $H_{q b}(R)^{+}$, then there are $f^{ \pm} \in$ $L^{1}(\partial R, d \omega)^{+}$such that $u^{ \pm}=H_{f^{ \pm}}^{R}$. Hence the choice $f:=f^{+}-f^{-} \in L^{1}(\partial R, d \omega)$ suffices to deduce (9.1) for $u$. Hence we can assume that $u \geq 0$. Next let $u_{i}:=u \wedge i \in H B(R)^{+}$for each $i \in N$. Then $u_{i} \uparrow u$ on $R$. If (9.1) is valid for functions in $H B(R)^{+}$, then there are $f_{l} \in L^{1}(\partial R, d \omega)$ such that $u_{i}=H_{f_{i}}^{R}(i \in N)$. By Proposition 2.1, $0 \leq f_{l} \leq i$ and $f_{l} \leq f_{l+1}(i \in N)$. Hence $f:=\lim _{i \rightarrow \infty} f_{l}$ exists and is $d \omega$-measurable on $\partial R$. Since $0 \leq \int_{\partial R} f_{l} d \omega=u_{i}\left(x_{0}\right) \leq u\left(x_{0}\right)$, we have $0 \leq \int_{\partial R} f d \omega=\lim _{i \uparrow \infty} \int_{\partial R} f_{l} d \omega \leq u\left(x_{0}\right)$, i.e. $f \in L^{1}(\partial R, d \omega)$. Hence $H_{f_{l}}^{R} \uparrow$ $H_{f}^{R}(i \uparrow \infty)$ and $H_{f_{i}}^{R}=u_{i} \uparrow u(i \uparrow \infty)$ assure the validity of $(9.1)$ for $u$. Thus we can assume that $u \in H B(R)^{+}$in proving (9.1). For $u \in H B(R)^{+}$there is a constant $c>0$ such that $0 \leq c u \leq 1$ on $R$. If $c u=H_{f}^{R}$ for an $f \in L^{1}(\partial R, d \omega)$, then $u=H_{c^{-1} f}^{R}$. Therefore, finally, we have seen that we may assume $0 \leq u \leq 1$ in proving (9.1).

Let $\left\{U_{i}\right\}_{0 \leq l \leq l}$ be an admissible covering of $\bar{R}$ and $\left\{\varphi_{i}\right\}_{0 \leq l \leq m}$ be an admissible partition of unity on $\bar{R}$ subordinate to $\left\{U_{i}\right\}_{0 \leq l \leq l}$ (cf. $\S 4$ ). For each $0 \leq i \leq m$ there exists a $j=j_{l}(0 \leq j \leq l)$ such that spt $\varphi_{i} \subset U_{J_{l}}$ with $j_{0}=0$ and $j_{l} \neq 0(1 \leq i \leq m)$. We set $V_{l}:=U_{j_{l}} \cap R(0 \leq i \leq m)$. By (5.1) and in fact trivially $u \in N(R)$ and by (5.2) we have $\varphi_{i} \in N(R)(0 \leq i \leq m)$. Since $N(R)$ is an algebra, $u_{i}:=u \varphi_{i} \in N(R)(0 \leq i \leq m) \quad(\mathrm{cf} . \S 4)$ and $u=u \cdot 1=u \sum_{l=0}^{m} \varphi_{i}=\sum_{l=0}^{m}$ $u \varphi_{i}=\sum_{l=0}^{m} u_{i}$ on $R$, i.e. we have

$$
u=\sum_{i=0}^{m} u_{i}
$$

with $u_{i} \in N(R)(0 \leq i \leq m)$ and $\left(u_{i}, V_{l}\right)$ plays the role of $(v, V)$ in $\S 6$ for each $i(1 \leq i \leq m)$. In view of $h_{u_{0}}^{R} \equiv 0$, we have

$$
u=h_{u}^{R}=h_{\sum_{i=0}^{m} u_{i}}^{R}=\sum_{l=1}^{m} h_{u_{i}}^{R}=\sum_{i=1}^{m} h_{u_{i}}^{V_{l}}+\sum_{l=1}^{m}\left(h_{u_{i}}^{R}-h_{u_{l}}^{V_{i}}\right) .
$$

Here $h_{u_{i}}^{V_{1}}$ are understood to be nonnegative continuous subharmonic functions on $R$ by setting $h_{u_{l}}^{V_{l}}=0$ on $R \backslash V_{l}$. By Lemma $6.1, h_{u_{t}}^{R}-h_{u_{i}}^{V_{l}} \in \mathscr{P}(R)(1 \leq i \leq m)$ and thus $P:=\sum_{l=1}^{m_{l}}\left(h_{u_{t}}^{R}-h_{u_{i}}^{V_{l}}\right) \in \mathscr{P}(R)$ and

$$
u=\sum_{l=1}^{m} h_{u_{i}}^{V_{l}}+P \quad \text { on } R .
$$


Since $h_{u_{i}}^{V_{l}} \in H\left(V_{l}\right)$ and, in view of Lemma 6.1, has boundary values zero on $\partial V_{l} \backslash U_{j_{l}}$, there is, by Lemma 8.1, a Borel function $g_{i}$ on $\partial V_{l}$ with $0 \leq g_{i} \leq 1$ and $g_{i}$ vanishes continuously at each point of $\partial V_{l} \backslash U_{j_{i}}$ such that $h_{u_{l}}^{V_{l}}=H_{g_{l}}^{V_{l}}$ on $V_{l}(1 \leq i \leq m)$. We set $f_{l}=g_{i}$ on $\bar{V}_{l} \cap \partial R$ and $f_{l}=0$ on $\boldsymbol{R}^{d} \backslash \bar{V}_{l} \cap \partial R$. Then $f_{l}$ is a Borel function on $\boldsymbol{R}^{d}$ with $0 \leq f_{l} \leq 1$ and $h_{u_{l}}^{V_{l}}=H_{f_{l}}^{V_{l}}$ on $V_{\imath}$ and hence on $R$ by setting $H_{f_{l}}^{V_{l}}=0$ on $R \backslash V_{l}(1 \leq i \leq m)$. Let $f:=\sum_{l=1}^{m} f_{l}$, which is a bounded nonnegative Borel function on $\partial R$. We have

$$
\begin{aligned}
\sum_{l=1}^{m} h_{u_{i}}^{V_{l}} & =\sum_{l=1}^{m} H_{f_{l}}^{V_{l}}=\sum_{l=1}^{m} H_{f_{i}}^{R}-\sum_{l=1}^{m}\left(H_{f_{i}}^{R}-H_{f_{l}}^{V_{l}}\right) \\
& =H_{\sum_{i=1}^{m} f_{l}}^{R}-\sum_{l=1}^{m}\left(H_{f_{l}}^{R}-H_{f_{i}}^{V_{l}}\right)=H_{f}^{R}-\sum_{t=1}^{m}\left(H_{f_{l}}^{R}-H_{f_{l}}^{V_{l}}\right)
\end{aligned}
$$

on $R$. Since $H_{f_{l}}^{R}-H_{f_{i}}^{V_{i}} \in \mathscr{P}(R)(i=1, \ldots, m)$ by Lemma 7.1 and thus $Q:=$ $\sum_{l=1}^{m}\left(H_{f_{i}}^{R}-H_{f_{i}}^{V_{l}}\right) \in \mathscr{P}(R)$, we have

$$
\sum_{l=1}^{m} h_{u_{t}}^{V_{l}}=H_{f}^{R}-Q \quad \text { on } R .
$$

By (9.2) and (9.3), we see that $u=H_{f}^{R}+P-Q$ on $R$, which implies that

$$
\left|u-H_{f}^{R}\right| \leq P+Q
$$

on $R$. Since the nonnegative subharmonic function $\left|u-H_{f}^{R}\right|$ on $R$ is dominated by the potential $P+Q$ on $R$, we must have $\left|u-H_{f}^{R}\right| \equiv 0$ on $R$, which proves (9.1).

\section{REFERENCES}

[1] C. Constantinescu UNd A. CoRnea, Ideale Ränder Riemannscher Flächen, Springer, 1963.

[2] L. L. Helms, Introduction to Potential Theory, Wiley-Interscience, 1969.

[3] Y. IshiKawa AND M. NAKAI, Regular and stable points in Dirichlet problem, Proc. Japan Acad. Ser. A Math. Scl., 73 (1997), 1-4.

[4] L. Sario AND M. NaKaI, Classification Theory of Riemann Surfaces, Springer, 1970.

(Professor Emeritus at)

Department of Mathematics

Nagoya Institute of TeChNology

GoKISO, SHOWA, NAGOYA 466-8555

JAPAN

(Mailing address)

52 Eguchi, Hinaga

CHITA 478-0041

JAPAN

e-mail: naka1@daldo-1t.ac.jp 